\title{
Research Paper \\ Comparing the Balance of Male Athletes Aged 11-14 Years With and Without Genu Varum
}

\author{
${ }^{*}$ Reza Hosseini ${ }^{1}$ (1), Ali Asghar Norasteh ${ }^{1}$, Nezam Nemati ${ }^{1}$
}

1. Department of Corrective Exercises and Sport Injuries, Faculty of Physical Education and Sport Sciences, University of Guilan, Rasht, Iran.

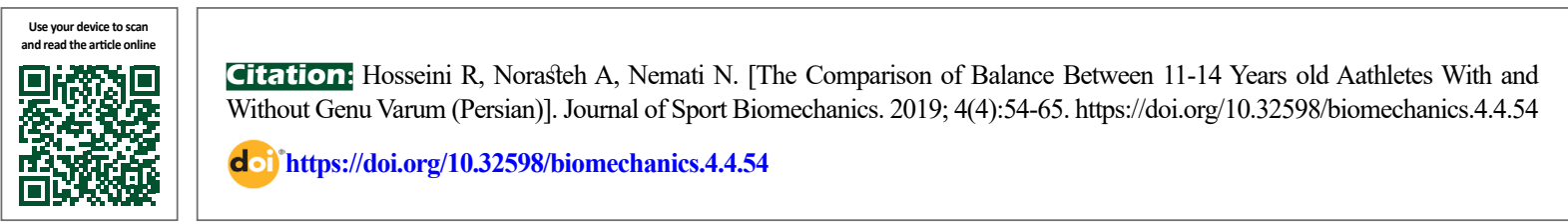

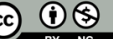

Article Info:

Received: 10 Nov 2018

Accepted: 28 Jan 2019

Available Online: 01 Mar 2019

Keywords:

Genu varum, Dy-

namic balance, Static

balance

\section{A B STRACT}

Objective Maintaining balance is one of the most important functions of neuromuscular system in performing all simple and complex $\neg$ sports activities. Musculoskeletal deformities especially, genu varum in the lower extremity, can negatively affect the body's biomechanics. The aim of this study was to the compare the balance of male athletes aged 11-14 years with and without genu varum.

Methods This study has a causal-comparative design. From a total of 580 amateur adolescent male athletes in basketball, handball and volleyball living in Marivan County, who had training three sessions per week, 21 with genu varum (Mean \pm SD of age=13.15 \pm 1.22 years; Mean $\pm S D$ of height $=1.68 \pm 2.32 \mathrm{~cm}$; Mean $\pm S D$ of weight $=51.23 \pm 4.48 \mathrm{~kg}$ ) and 21 with no genu varum (Mean $\pm S D$ of age $=12.95 \pm 1.17$ years; Mean $\pm S D$ of height $=1.67 \pm 1.45 \mathrm{~cm}$; Mean $\pm S D$ of weight $=49.49 \pm 3.48 \mathrm{~kg}$ ) were recruited. Their genu varum deformity was assessed using a caliper. Static and dynamic balance was evaluated by Bass Stick test and $Y$ Balance test, respectively. Data analysis was performed using independent samples t-test.

Results There was no significant difference in static balance between those with and without genu varum $(P=0.61)$, while their dynamic balance was significantly different in posteromedial $(P=0.003)$ and posterolateral $(P=0.004)$ directions.

Conclusion Negative effect of knee deformity on balance are turned out over time. Preventive measures and appropriate exercises at an early age may reduce the negative effects of deformities on balance.

\section{Extended Abstract}

\section{Introduction}

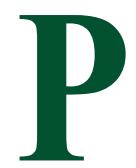

ostural control is defined as the control of body position in space to maintain body stability and orientation. Postural orientation is the ability to maintain a proper connection between body segments as well as between the body and the environment to perform a task. In many functional tasks, body alignment is vertical.

Furthermore, to maintain this state, it applies various sensory stimuli, such as gravity (vestibular system), the base of support (somatosensory system), and the body's relationship to objects in the environment (visual system). The lower extremity, due to its significant role in enduring weight, absorbing and modifying the pressures and kicks during dynamic activities (e.g. walking, running, jumping),

\section{* Corresponding Author:}

Reza Hosseini, Msc.

Address: Department of Corrective Exercises and Sport Injuries, Faculty of Physical Education and Sport Sciences, University of Guilan, Rasht, Iran Tel: +98 (918) 8745003

E-Mail: rezahosseini1196aran@gmail.com 
and maintaining the postural control in standing and moving positions, is of particular importance [2]. The knee joint plays a significant role in supporting the body and transmitting its weight during static and dynamic activities; however, since it has almost no bone component to stabilize it, it is one of the most vulnerable joints in the lower extremity [3].

Genu varum is among the knee deformities [4]. Such changes in the lower limb can disturb the center of gravity relative to the base of support; they might ultimately cause significant changes in individuals' balance. The mechanical axis of the knee usually passes through the center of the knee joint, i.e. from the tubercle between the tibial condyle, and when standing on two legs, the force is equally distributed between the inner and outer knee parts [5]. Genu varum deformity affects the mechanical axis deviation of the knee joint. Moreover, internal and external ankle rotation influences the mechanical axis deviation of the ankle joint. Therefore, the mechanical axis deviation of the lower extremity joints could significantly impact on the forces exerted by the ground as well as balance disturbance [6]. Researchers disregarded investigating the effects of genu varum deformity on the balance of athletes at younger ages. Thus, this study aimed to investigate the static and dynamic balance of adolescent athletes with and without genu varum deformity.

\section{Participants and Methods}

A total of 580 adolescent male athletes aged 11-14 years in Marivan County, Iran, were screened for genu varum deformity using a caliper. Of them, 42 with and without genu varum were purposively selected as the study samples. The study participants were divided into 2 groups of 21 .

To detect genu varum, the subject stood bare feet on both legs without any contractions and abnormal tonicity in the thigh muscles. The knees were in full extension and the ankles stick together where the patella bones were facing

Table 1. Mean \pm SD scores of study variables forward. In this state, the distance between the two medial femoral condyles was measured by a caliper. A distance of $>3 \mathrm{~cm}$ between the two condyles was considered as a genu varum [5]. The Bass Stick test was used to measure the static balance of the study subjects [14]. Besides, the Y Balance Test (YBT) was used to evaluate the dynamic balance $[15,16]$.

\section{Results}

Table 1 presents Mean \pm SD values of the study variables. The t-test results (Table 2) suggested no significant difference in static balance between the study subjects with and without genu varum. Moreover, the results dynamic balance test in anterior direction revealed no significant difference between the study groups; however, group differences were significant in posterolateral $(\mathrm{t}=3.15, \mathrm{P}=0.004)$ and posteromedial $(\mathrm{t}=3.17, \mathrm{P}=0.003)$ directions. In other words, the study subjects with genu varum had less dynamic balance in lateral posterior and medial posterior directions, compared to their healthy counterparts.

\section{Discussion}

In this study, no statistically significant difference was found in the static balance between the study subjects with genu varum and healthy controls. This could be because the sport has a dynamic nature, rather than a static one; thus, it did not affect the static balance of subjects. In dynamic balance, the difference between the two study groups was only significant in the anterior direction. In other words, athletes with genu varum had less dynamic balance than their healthy peers in lateral posterior and medial posterior directions. The obtained static balance test data was in line with some other studies. Shojaedin et al. [10] investigated the relationship between varus knee deformity and dynamic and static postural control in 10-12-year-old boys. Their results indicated that varus knee deformity did not affect the samples' static balance.

\begin{tabular}{|ccc|}
\hline & & Mean \pm SD \\
Variable & Without Genu Varum & With Genu Varum \\
\cline { 2 - 3 } & $1.29 \pm 0.42$ & $1.23 \pm 0.25$ \\
\hline Static balance (s) & $81.76 \pm 12.63$ & $79.64 \pm 6.42$ \\
\hline Dynamic balance (\%) & $69.98 \pm 11.19$ & $72.58 \pm 9.18$ \\
\hline Anterior direction (\%) & $75.29 \pm 13.16$ & $65.33 \pm 10.96$ \\
\hline Lateral posterior direction (\%) & $75.15 \pm 12.90$ & $64.46 \pm 7.81$ \\
\hline Medial posterior direction (\%) & & Journal of \\
\hline
\end{tabular}


Table 2. Levene's test and t-test results for dynamic and static balance measurements

\begin{tabular}{|c|c|c|c|c|c|c|}
\hline \multirow{2}{*}{ Variable } & \multicolumn{2}{|c|}{ Levene's Test } & \multicolumn{3}{|c|}{ t-test } & \multirow{2}{*}{$\begin{array}{c}\text { Mean Difference } \\
\text { P<0.01** }\end{array}$} \\
\hline & $\mathbf{F}$ & Sig. & $\mathbf{t}$ & df & Sig. & \\
\hline Static balance (s) & 2.64 & 0.12 & 0.514 & 38 & 0.61 & 0.056 \\
\hline Anterior direction (\%) & 0.267 & 0.69 & 0.803 & 38 & 0.42 & 2.60 \\
\hline $\begin{array}{l}\text { Lateral posterior direc- } \\
\text { tion }(\%)\end{array}$ & 0.855 & 0.36 & $3.15^{* *}$ & 38 & 0.004 & 9.96 \\
\hline $\begin{array}{l}\text { Medial posterior } \\
\text { direction (\%) }\end{array}$ & 0.853 & 0.361 & $3.17^{* *}$ & 38 & 0.003 & 10.68 \\
\hline
\end{tabular}

\section{Conclusion}

Varus knee deformity could affect the dynamic balance of athletes compared to their healthy counterparts. The adverse effects of such deformities on balance increases with aging. It is recommended that this study be conducted with a larger sample size as well as a more extensive age population range to be able to generalize the findings to different groups.

\section{Ethical Considerations}

Compliance with ethical guidelines

Prior to the study, a brief explanation of the tests and methods were given to the participants. Then, a written informed consent was obtained from their parents and they were told that they were to leave the study at any time. Moreover, permissions were obtained from the Department of Youth Affairs and Sports in Marivan, Iran.

Funding

This research did not receive any financial support from funding agencies in the public, commercial, or not-for-profit organizations.

\section{Authors' contributions}

Investigation and draft preparation: Reza Hosseini; review and editing: Ali Asghar Norasteh and Nezam Nemati.

Conflicts of interest

The authors declare no conflict of interest. 


\title{
مقايسه تعادل ورزشكاران || تا ع ( سالِ باو بدون زانوى يرانتزى
}

\author{
'رضا حسينى' ـ، علىاصغر نورسته'، نظام نعمتى' \\ ا. كروه آسيبشناسى ورزشى و حركات اصلاحي، دانشكده تربيتبلنى و علوم ورزشي، دانشكاه كيلان، رشت، ايران.
}

\begin{abstract}
حكيد

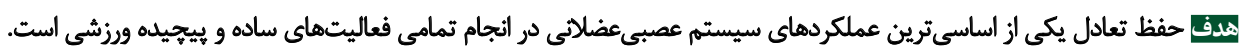

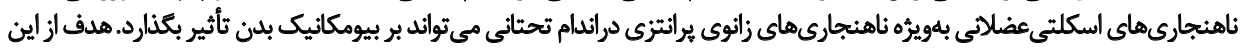

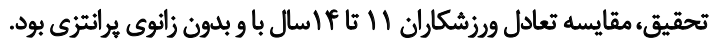

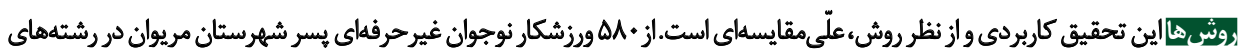

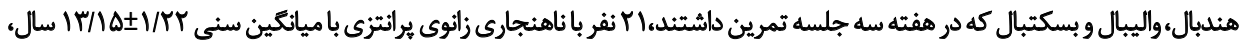
قد وز

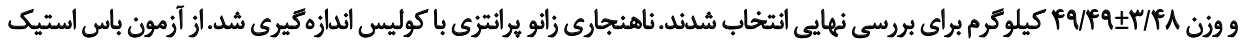

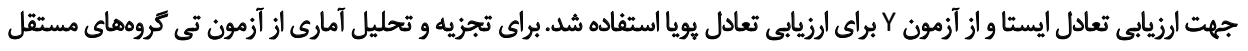

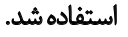

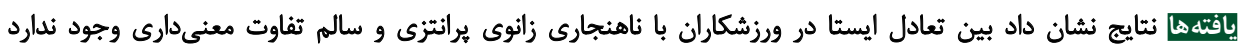

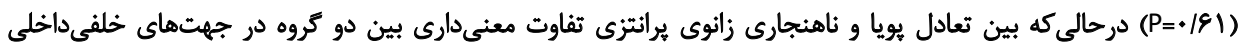

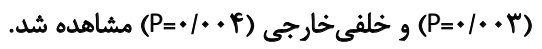

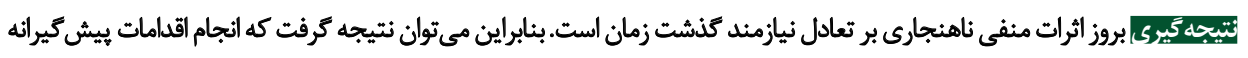

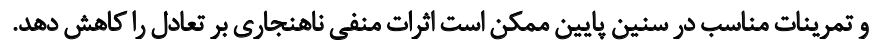

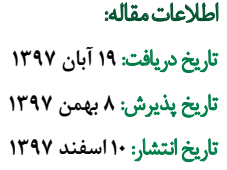

كليدواروها: زائري

زانوى برائزي، تعادل

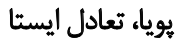

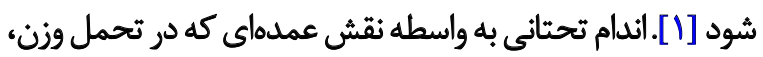

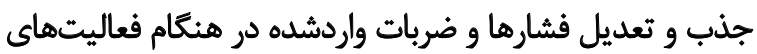

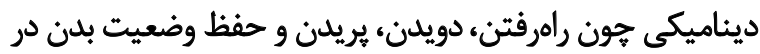

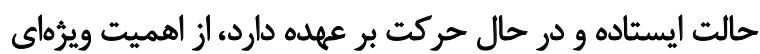

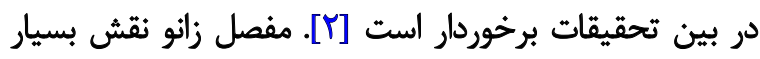

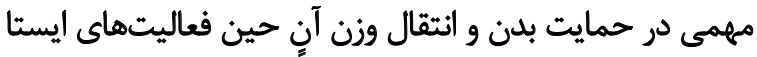

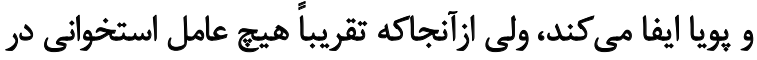

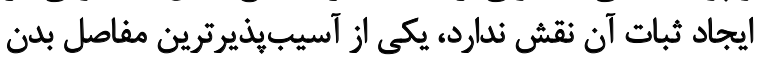

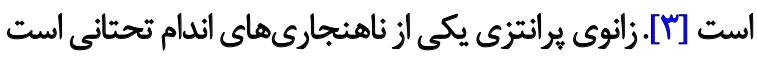

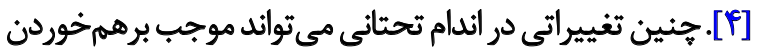

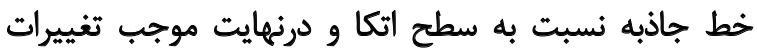

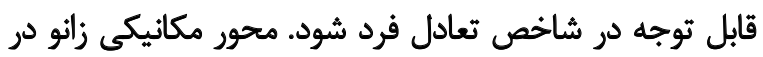

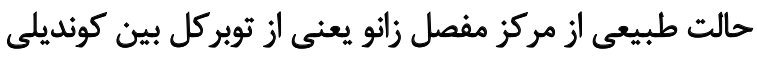

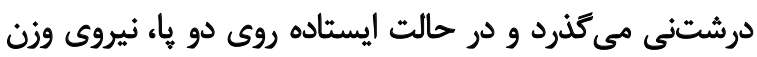

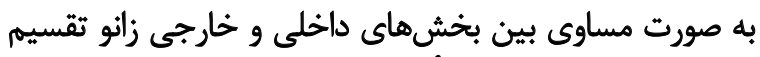

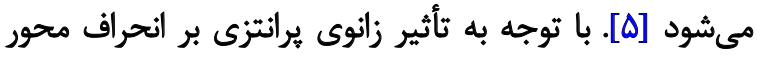

كنترل ياسجر به صورت كنترل وضعيت بلن در فضا به منظور

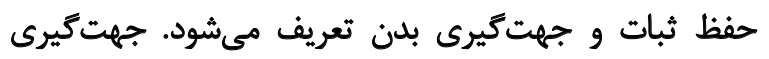

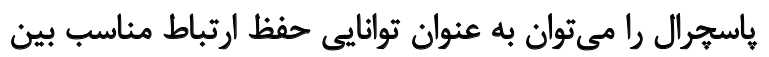

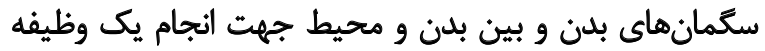

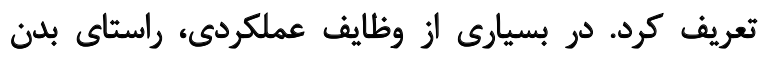

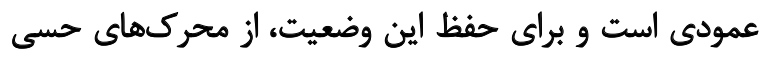
كوناكون همجيون جاذبه (سيستم وستيبولار)، سطح اتكا (سيستم حسي يويكرى) و ارتباط بدن با اشياى موجود در محيط (سيستم بينايى) استفاده مي شود.

ثبات باسجرال يا تعادل، توانايى بدن در حفظ توازن بين نيروها

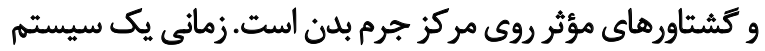

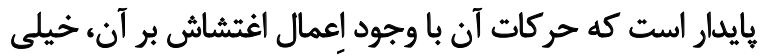

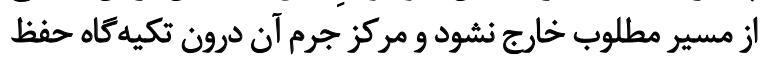

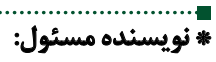

$$
\begin{aligned}
& \text { رضا حسينى } \\
& \text { نشائى: رشت، دانشعاه كيلان، دانشكده تربيثبدنى و علوم ورزشى، كروه آسيبشناسى ورزشى و حركات اصلاحى. }
\end{aligned}
$$

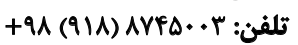


ورزشكار بر روى كنترل تعادل بدن وجود دارد. زانو يكى از مفاصل

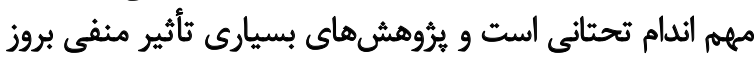

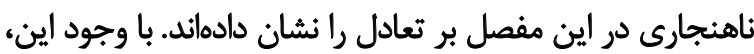

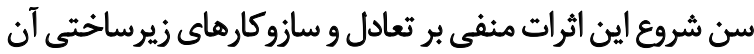

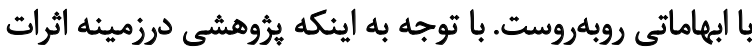

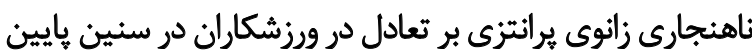

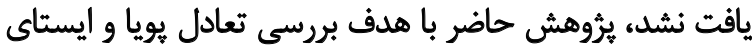

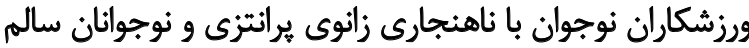
طراحى شده است.

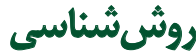

با توجه به اهداف و محتواى تحقيق حاضر، اين تحقيق كاربردى

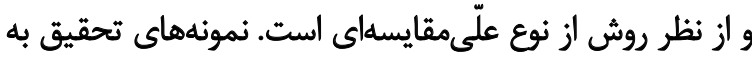

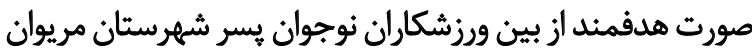

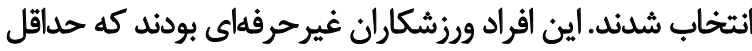

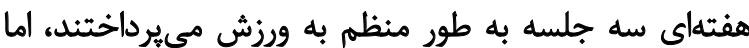

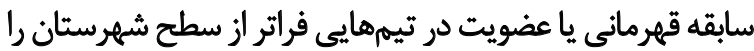

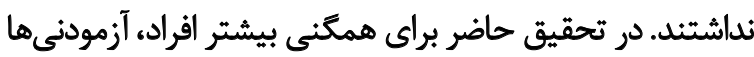

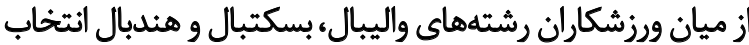

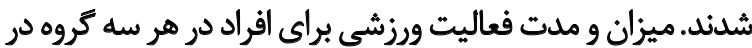

سطح يكسانى بود [1/1]].

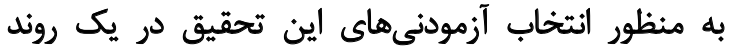

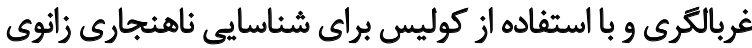

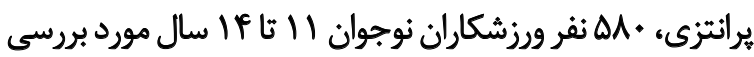

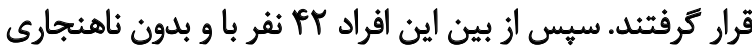

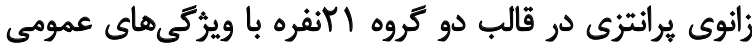

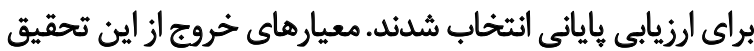

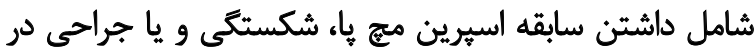

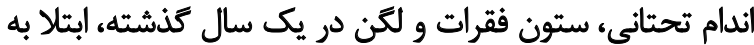

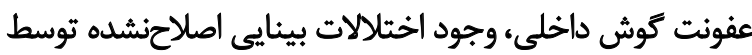

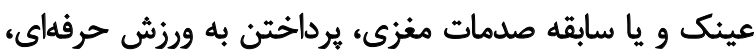

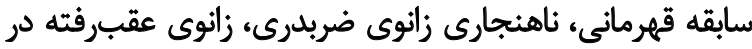

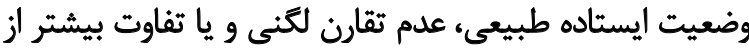

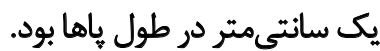

روش اندازهيّي ز أنوى برانتزى با استفاده از كوليس

براي اندازهيرى اين ناهنجارى، فرد بدون كفش و جوراب

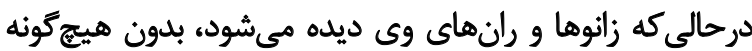

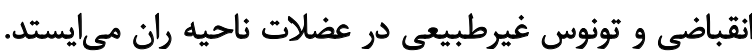

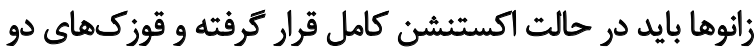

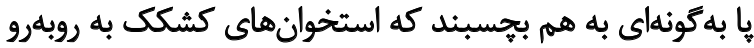

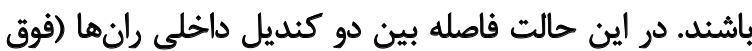

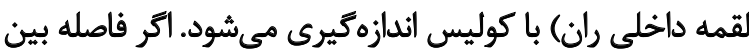

مكانيكى مفصل زانو و همينطور تأثير جرخش داخلى وخاني وخارجى

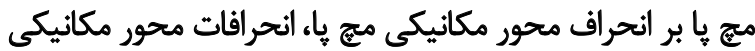

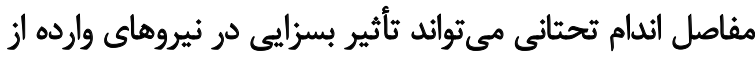

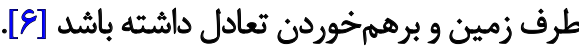
در سالهاى اخير در تحقيقات به بررسى تأثير ناهنجارى ئري

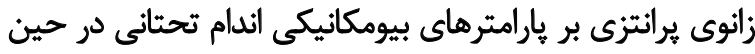

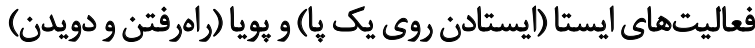

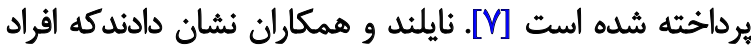

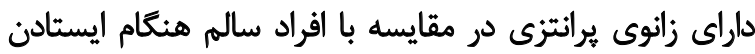

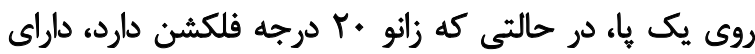

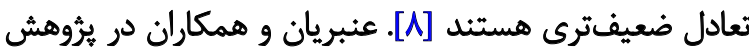

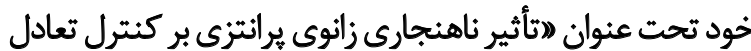

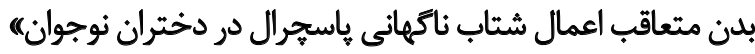

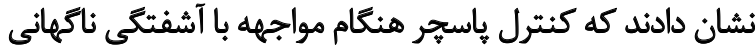

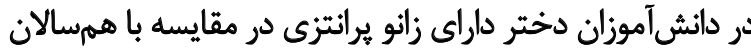

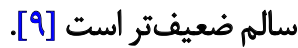

بختيارى و همكاران در تحقيق خود تحت عنوان (ضاثر ناهنجارى

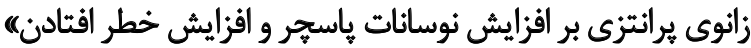

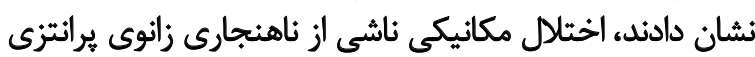

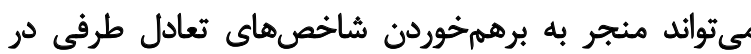

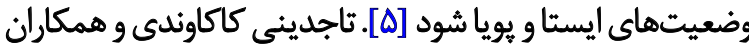

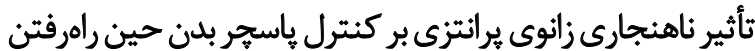

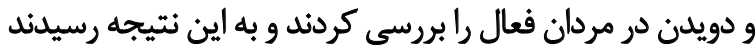

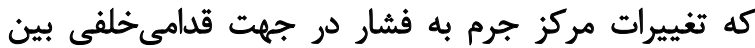

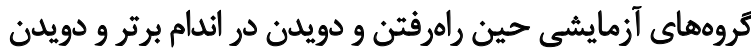

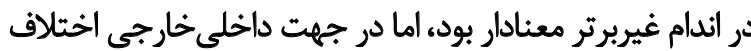

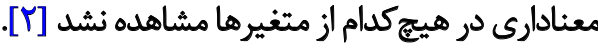

شجاعالدين و همكاران برخلاف يافتههاي تحقيقات قبلى نشان

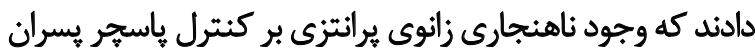

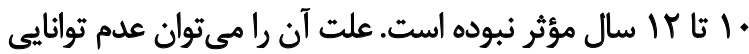

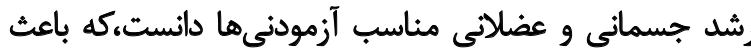

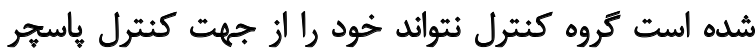

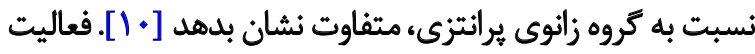

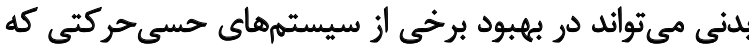

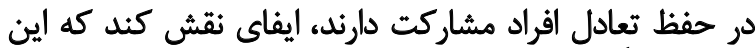

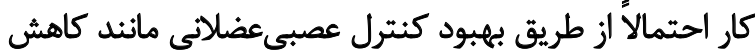

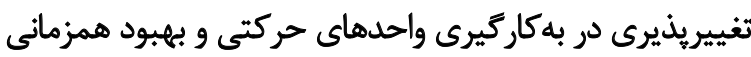
واحدهاى حركتى باشد [11].

با تأكيد بر تئورى سيستمها مشخص مى شود كه سيستمهاى

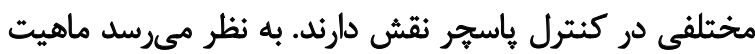

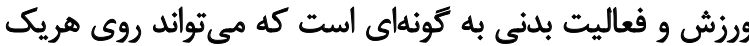

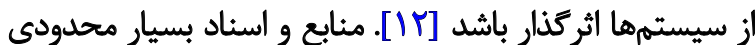

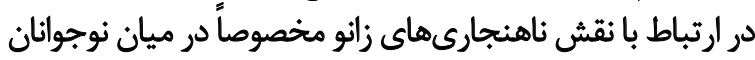




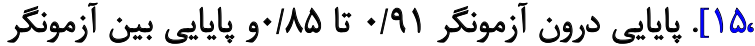

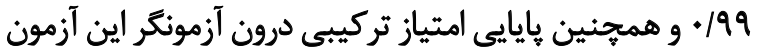

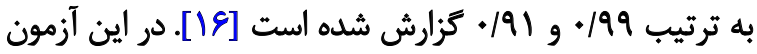

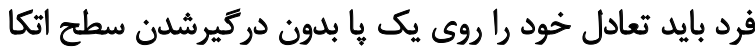

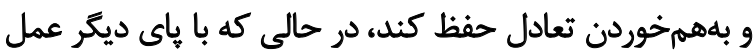

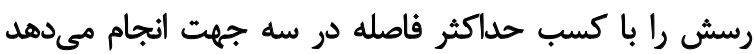

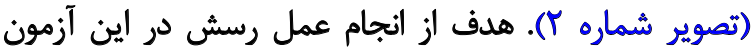

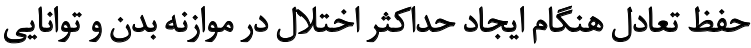

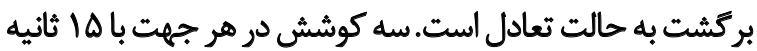

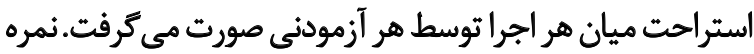

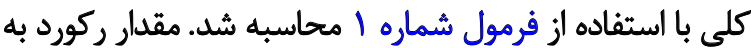

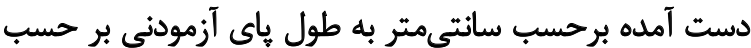

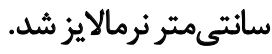

.1

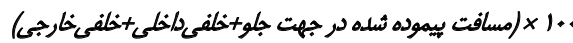
أميازتركيييق

براى تجزيه و تحليل اطلاعات جمعآورى شده از روشهاي آمارى

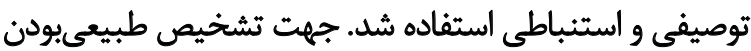

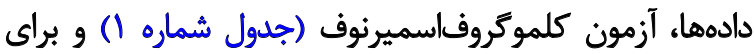

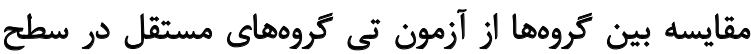

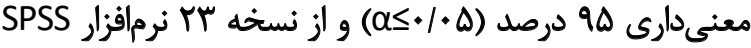

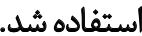

ثنتايج

جدول شماره 1 نتايج آزمون كلموموكروفاسميرنوف براى

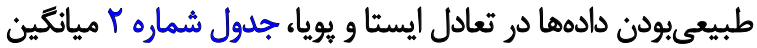

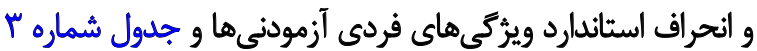

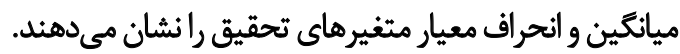
نتايج آزمون تى كروههاى مستقل (جدول شماره fi) نشان داد

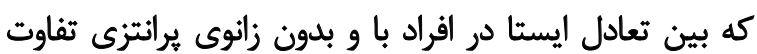

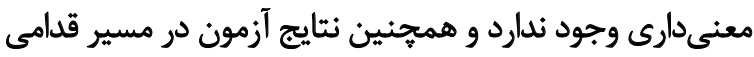

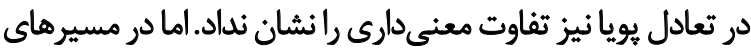

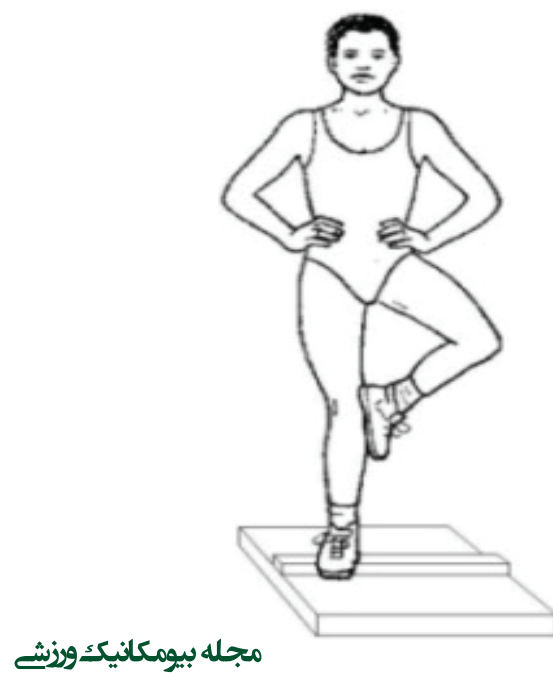

$$
\text { تصوير ا. نحوى ارزيابى تعادل ايستا }
$$

دو كنديل بيشتر از سه سانتىمتر باشد، زانوء برانتزى محسوب هىشود [ه]. روش ارزيابى تعادل ايستا

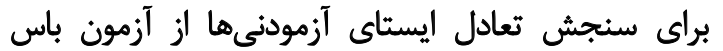

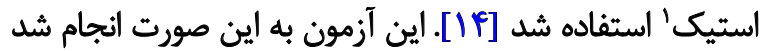

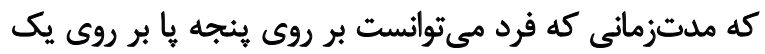

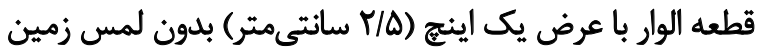

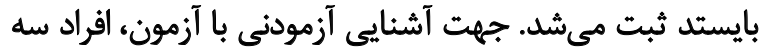

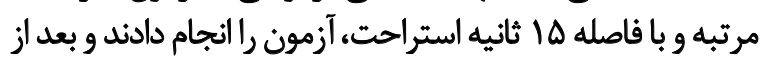

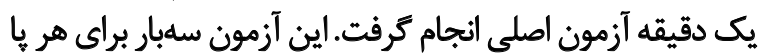

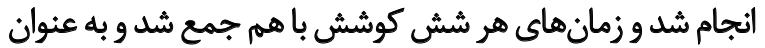
امتياز كلى در نظر كرفته شد (تصوير شماره لمان ).

$$
\text { روش الرزيابى تعادل يويا }
$$
19 از آزمون تعادل Y' براى ارزيابى تعادل يويا استفاده شد

1. Bass stick

2. Y Balance Test

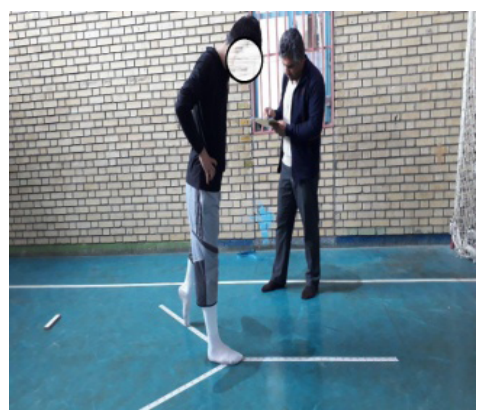

مجله بيومكانيك ورنثـ
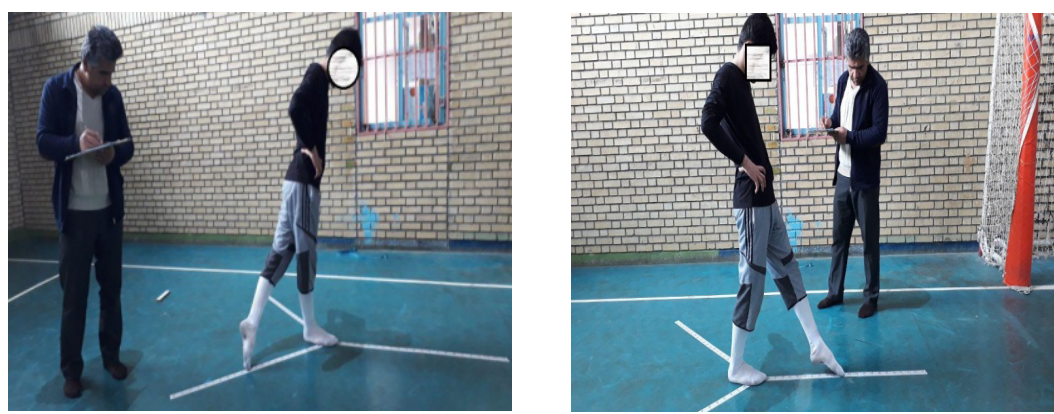

تصوير Y. نحوى ارزيابى تعادل يويا در مسيرهاي قدامى، خلفى خارجى وخلفى داخلى 
جدول اء آزمون كلوموكروف اسميرنوف براى طبيعى بودن دادهها در تعادل ايستا و يويا

\begin{tabular}{|c|c|c|c|}
\hline مقدار آزمون آمارى & تعداد & سطح معنيدارى & تعادل \\
\hline.$/ 1 P q$ & Mt &.$/ . \Delta V$ & إيسثا \\
\hline ./Ira & RT & .1 .99 & يويا \\
\hline$.|| f \mid$ & m &.$/ . \Delta V$ & قدامى \\
\hline$+M+r$ & m & $. / r+*$ & خلفى - خارجى \\
\hline.$/ 1 F V$ & HT & $\%$ & خلفى -ماخلى \\
\hline
\end{tabular}

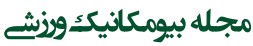

جدول Y . مربوط به ويرُكى هاى عمومى شركت كنيدكان

\begin{tabular}{|c|c|c|c|c|c|}
\hline \multicolumn{4}{|c|}{ مياتكين ثانحر اف معيار } & \multirow[b]{2}{*}{ كروه } & \multirow{2}{*}{ رشته ورزشى (تعداد } \\
\hline 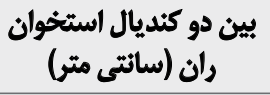 & وزن (كيلوكرم) & سن (سال) & قد (متر) & & \\
\hline$\Delta / 1 \cdot \pm \cdot / V \lambda$ & D)/RrEte/RA & $I M / / \Delta \pm I / T r$ & $V / E N \pm T / M T$ & زانو يرانتزى & \multirow{2}{*}{ واليبال (n=|" } \\
\hline$\cdot / 8 \Delta \pm+/ M$ & $F N \mid N \pm \Psi / F A$ & $\mid r / / Y \pm \| / M$ & $V / \mathscr{R} \pm T / \cdot r$ & سالم & \\
\hline$\Delta / F V \pm+189$ & $\Delta r / r \pm r / Q$ & $I r / \Lambda \cdot \pm 1 / T T$ & $V / F E \pm \mid / T I$ & زانو يرانتزى & \multirow{2}{*}{ بسكتبال (n=1f) } \\
\hline $1 / \cdot \Delta \pm \cdot / \Delta E$ & $\Delta \cdot / 1 Y \pm Y / \Delta V$ & $\mid r / \leftarrow \wedge \pm 1 / M \Lambda$ & $1 / 89 \pm 1 / 1 \%$ & سالم & \\
\hline$\Delta / \Delta \Delta \pm V / \cdot V$ & Fq/THET/HT & $|r / r \Delta \pm \| /|$ & V/gatr/mr & زانو يرانتزى & \multirow{2}{*}{ هندبال (n=11) } \\
\hline$\cdot / A V \pm \cdot / e q$ & $\Delta \cdot / N \pm T / R r$ & $\mid r / T V \pm V / 10$ & $N / N \cdot \pm 1 / M$ & سالم & \\
\hline
\end{tabular}

مجله بيومكانيك وزنث

زانوى يرانتزى و كروه افراد سالم مشاهده نشد. در مورد تعادل

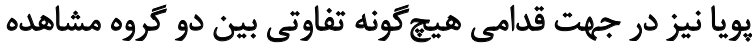

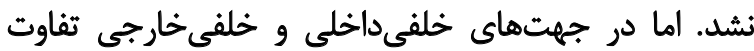

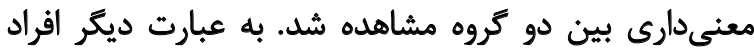

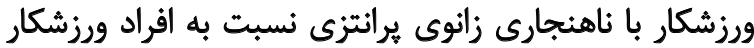
سالم در اين جهتها از تعادل كمترى داشتئندي تعادل نسادل كه

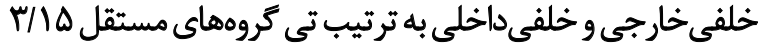

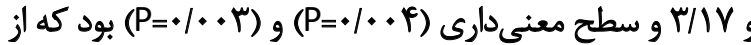

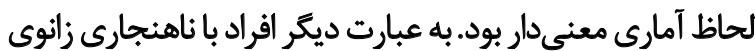

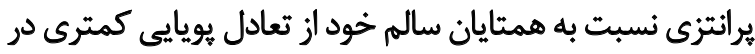
مسيرهاى خلفى خارجى وخلفى داخلى برخوردار بودند.

بحث

در اين يُوهش تفاوتى در تعادل ايستا بين كروه افراد داراى

\begin{tabular}{|c|c|c|}
\hline \multicolumn{2}{|c|}{ ميائكين+انحراف معيار } & \multirow{2}{*}{ متغير } \\
\hline داراى ناهنجارى زانويرانتزى & سالم & \\
\hline I/TY士+/TA & 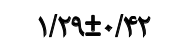 & تعادل ايستا(ثانيه) \\
\hline$V Q / g F \pm g / e r$ & A)/VEIIr/gr & تعادل يويا (درصدكلى) \\
\hline$V T / \Delta A \pm Q / M$ & $89 / 4 \pm 11 / 19$ & قدافي( درصد) \\
\hline $8 \Delta / r T \pm 1 \cdot / Q$ & Va/rq土ir/le & خلفي - خارجي( درصد ) \\
\hline$e f / e q \pm V(\lambda)$ & $V \Delta / 1 \Delta \pm 1 Y / 9$ & خلفى - داخلى(درصد) \\
\hline
\end{tabular}

مجله بيومكانيك ورنش 
جدول F. نتايج آزمون لون و تى كروههاي مستثل براي متغير ايستا و يويا

\begin{tabular}{|c|c|c|c|c|c|c|}
\hline \multirow{2}{*}{ اختلاف ميانكين } & \multicolumn{3}{|c|}{ أزمون معنى دارى تى كروههاى مستقل } & \multicolumn{2}{|c|}{ آزمون لون براي برابرى واريانسها } & \\
\hline & Sig. & DF & $\mathbf{t}$ & Sig. & $\mathbf{F}$ & \\
\hline $.1 . \Delta C$ & .181 & rA &.$(01)$ &.$/ r$ & r/ge & تعادل ايستا \\
\hline$r / 9$. & . Rer & rA & $\cdot / A \cdot Y$ & .189 & . ITEY & قدامى \\
\hline$q / 2$ & $.10 .+$ & $\mathrm{MA}$ & $H / / \Delta^{* * *}$ &.$/ r g$ &.$/ 1 A \Delta \Delta$ & خلفى خارجى \\
\hline $1.18 \mathrm{~A}$ & 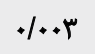 & rג & $r / / V * *$ &.$/ 481$ & - /Aar & خلفى داخلى \\
\hline
\end{tabular}

مجلله بيومكانيك ورنث

تفاوتى در عملكرد تعادل ايستا اين گروه از نوجوانان رانشان نداد.

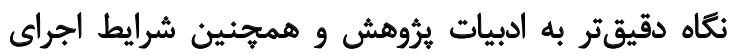

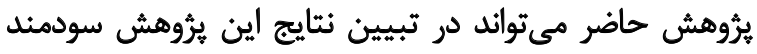

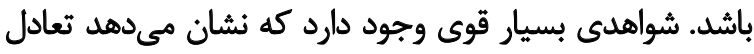

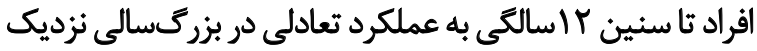

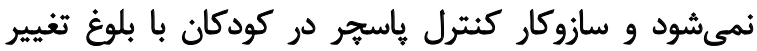

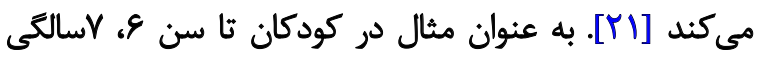

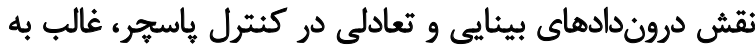

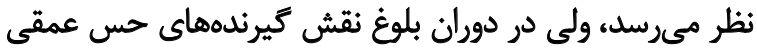

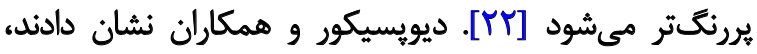

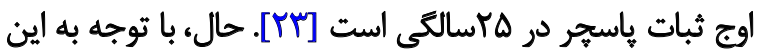

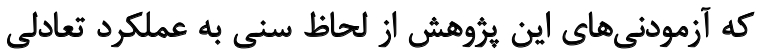

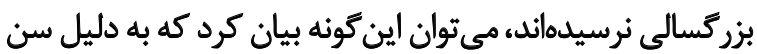

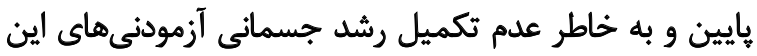

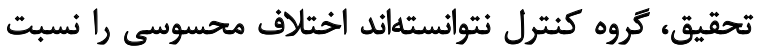

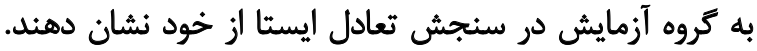

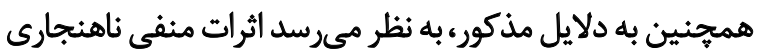

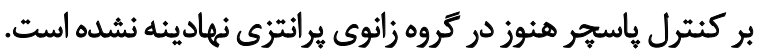

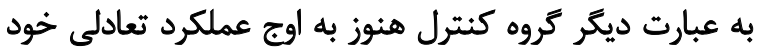

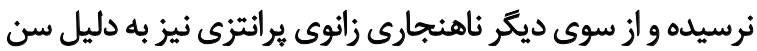

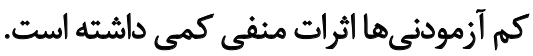

صادقي و همكاران به اين نتيجه رسيدند كه نوسان ياسجر

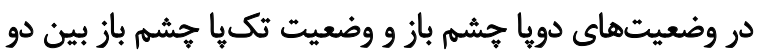

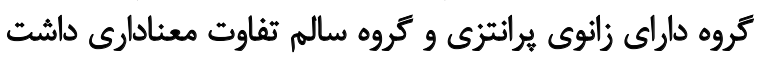

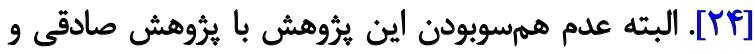

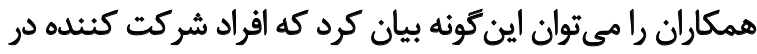

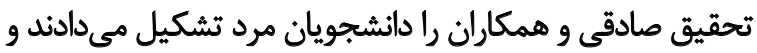

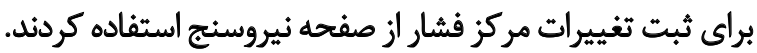

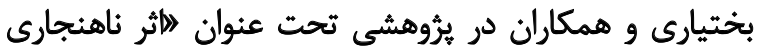

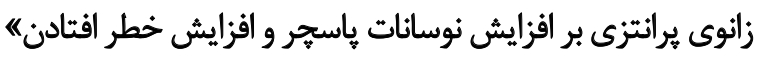

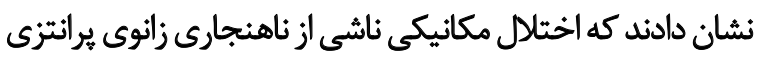
مىتواند منجر به برهمخوردن شاخصهاني ناشي تعادل طارئ طرفى دري
** سطح معنى دارى در اين يُروهش يك درصد است.

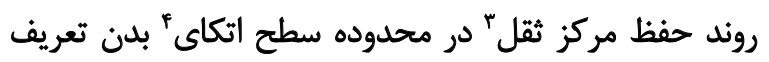

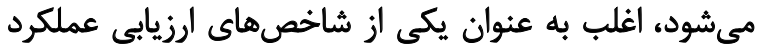

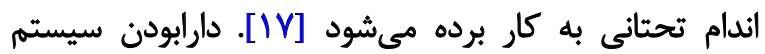

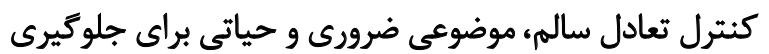
از آسيب هنكام فعاليتهاى روزمره است [11]

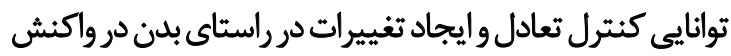

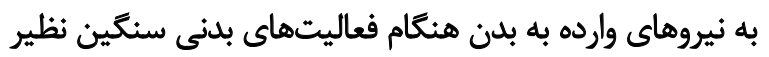

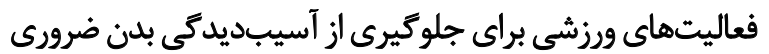

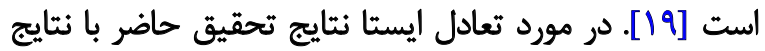

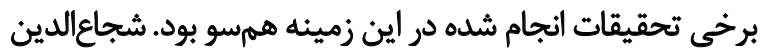

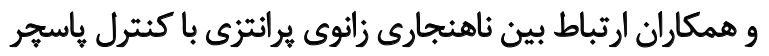

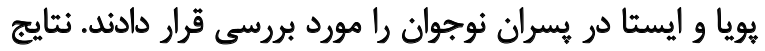

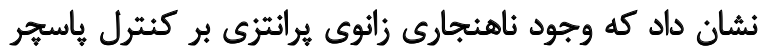

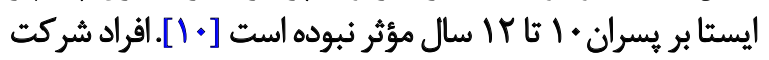

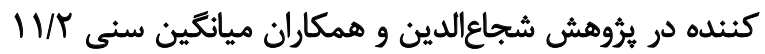

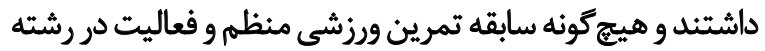
ورزشى خاص رانداشتند.

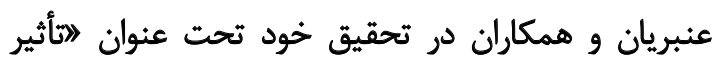

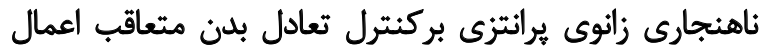

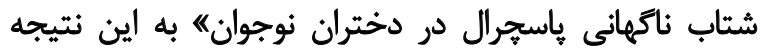

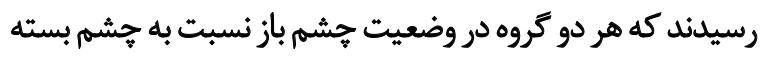

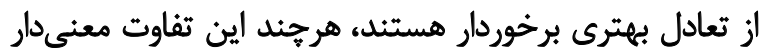

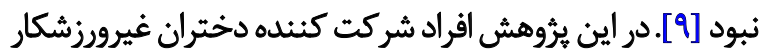

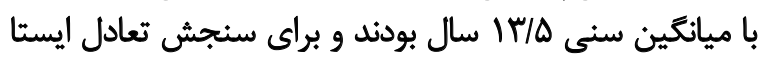

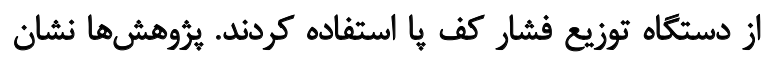

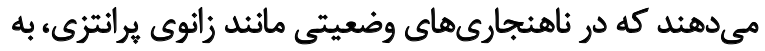

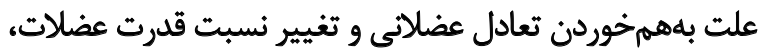

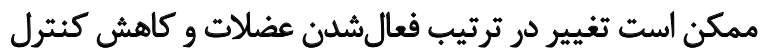

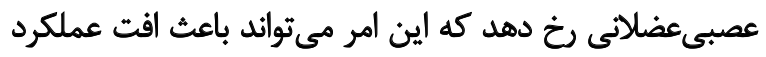

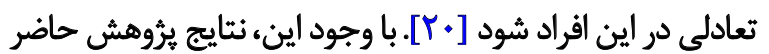

3. Center of gravity

4. Base of support 
براي افراد بيمار و مسن باشند، اما براى ارزيابى عملكرد تعادلى در

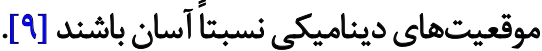

استيف و همكاران در تحقيقى بر روى تجزيه و تحليل راهرفتن

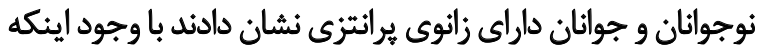

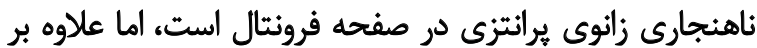

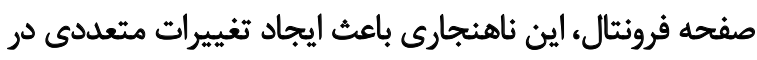

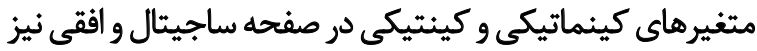

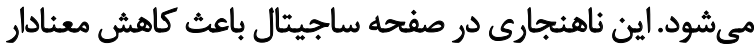

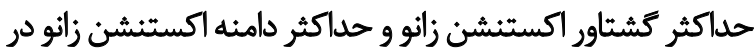

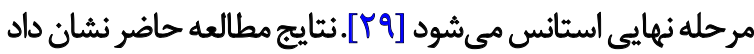

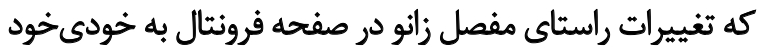

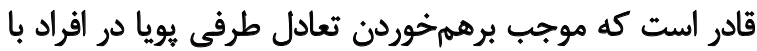

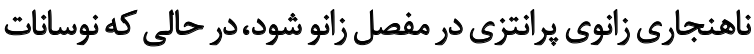

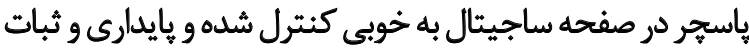
فرد دستخوش اختلال نشده است.

با توجه به تغيير راستاى ثاندون عضله جهار سر در افراد داراى

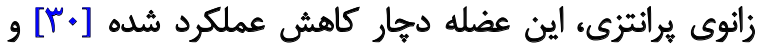

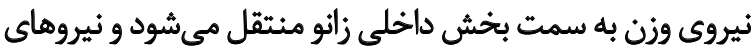

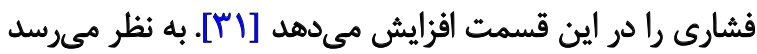

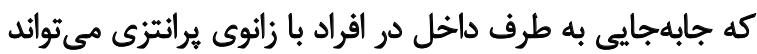

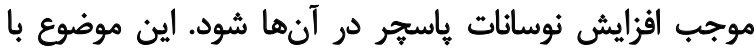

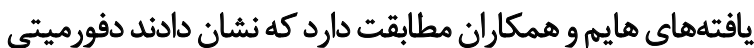

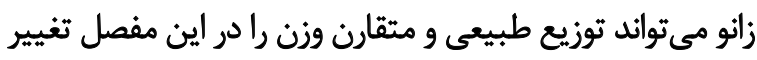

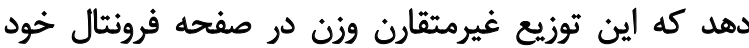

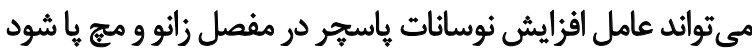

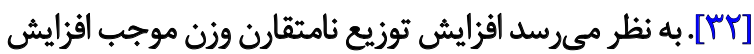

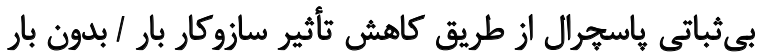

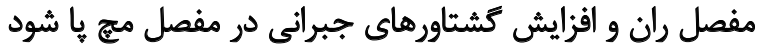

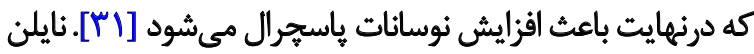

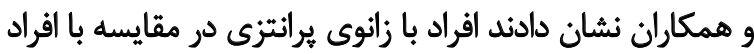

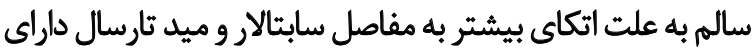

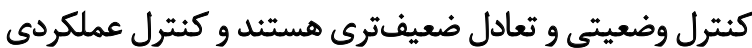

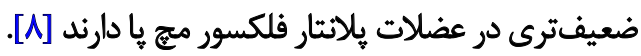

\section{نتيجهيَيرىنهايى}

نتايج يرؤهش حاضر نشان داد تعادل ايستاى افراد با ناهنجارى نائ

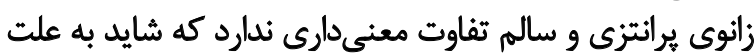

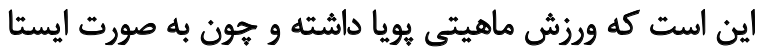

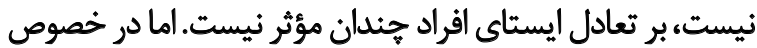

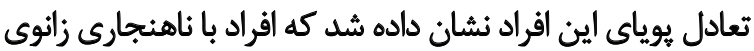

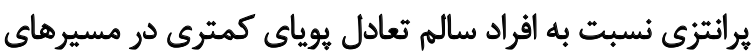

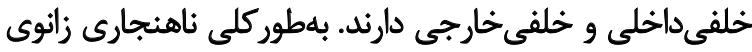

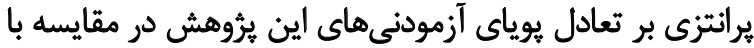

وضعيتهاى ايستا و يويا شود [ه]. اين اختلاف در نتايج بين

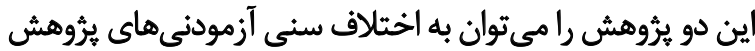

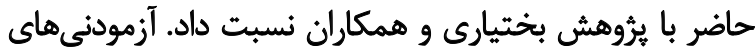

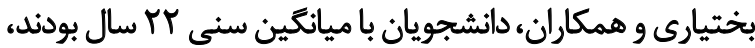

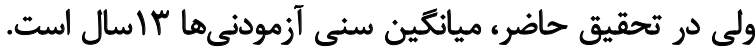

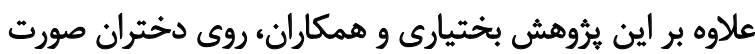

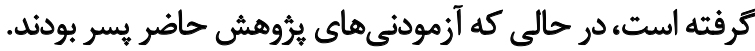

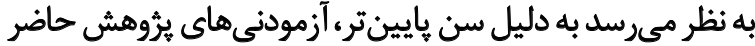

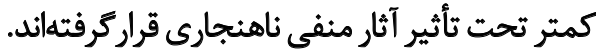

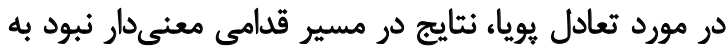

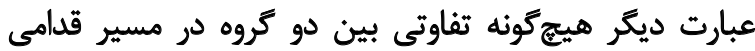

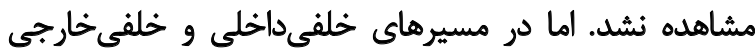

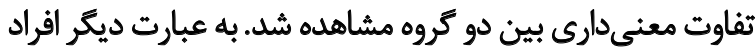

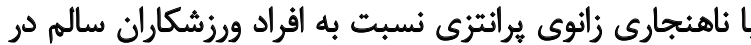

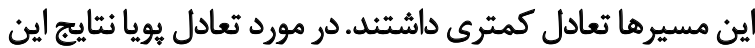

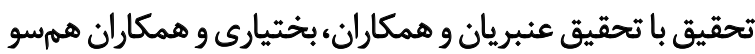

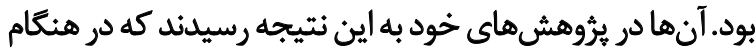

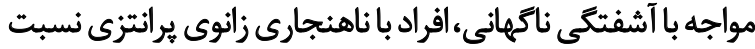

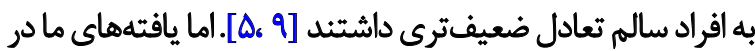

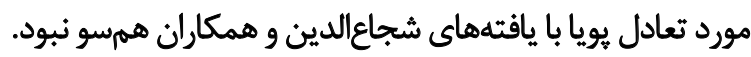
بر اساس نظريه سيستمها، حفظ تعادل و متعاقب آن ايجاد

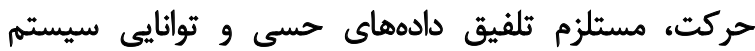

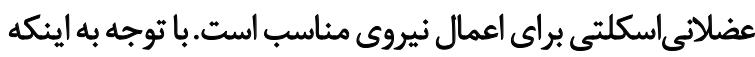

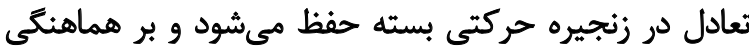

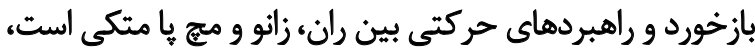

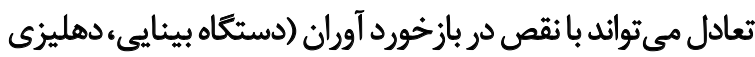
و حسى ييكرى) يا نقص در قدرث و و وإيدارى مكانيكيى هر مفصل

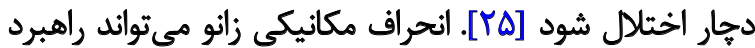

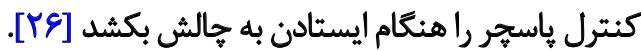

نورسته و همكاران نشان دادند كه اكر تقارن عضلات و موقعيت

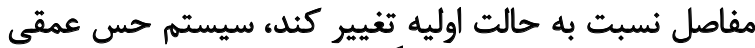

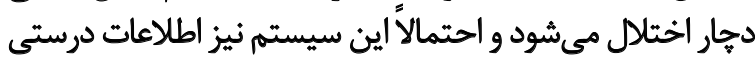

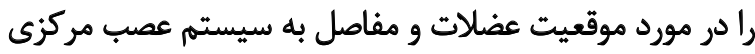

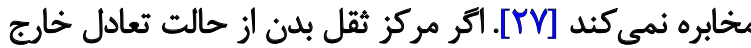

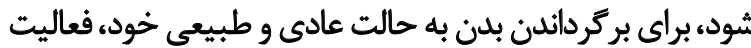

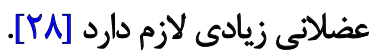

زمانى كه تعادل يوياى بدن در اثر حركات انثقالى يا رسشى ئري

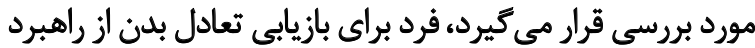

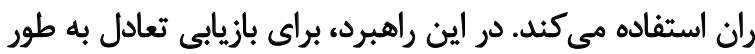

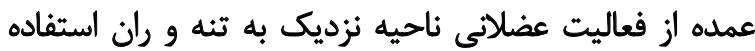

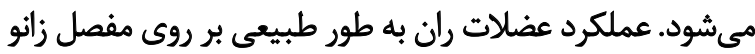

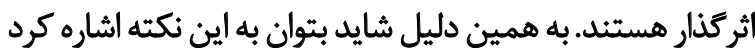

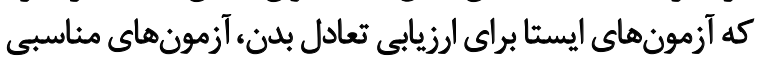




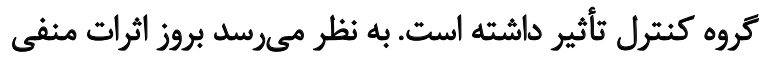

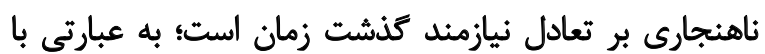

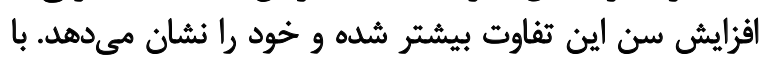

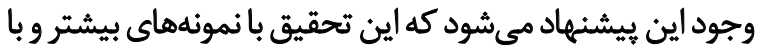

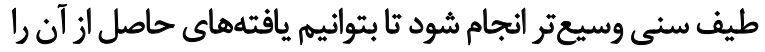
به كروههاى بيشتر تعميم دهيم. مالاحظات اخلاقى يبيروى از اصول اخلاق بثوهش قبل از شروع يُروهش، شح مختصرى در مورد نحوى اجراى

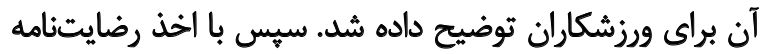

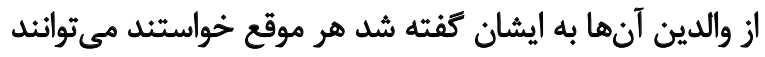

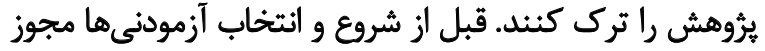

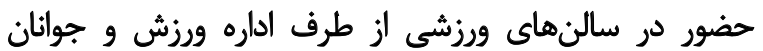

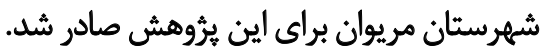

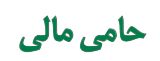

ايــن مقالـه از هيجيجونـهـ حمايــت مالـى برخــوردار نبـوده اسـت. ت است معات

$$
\text { مشاركت نويسندكَّان }
$$

جمعآورى دادهها و نكارش كليه بخشها: رضا حسينى؛

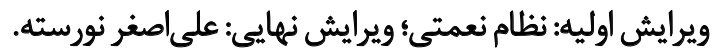

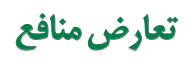

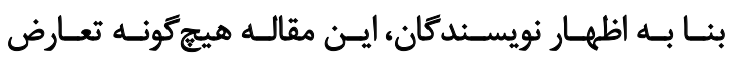
منافعى نداشـته اسـت 


\section{References}

[1] Taheri M, Irandoust Kh, Norasteh AA, Shavikloo J. [The effect of combined core stability and neuromuscular training on postural control in students with congenital hearing loss (Persian)]. Journal of Research in Rehabilitation Sciences. 2017; 13(2):80-6.

[2] Tajdini Kakavandi H, Sadeghi H, Abbasi A. [The effect of genu varum deformity on posture control during walking and running in active male (Persian)]. Journal of Applied Exercise Physiology. 2018; 14(27):65-76. [DOI:10.22080/JAEP.2018.1795]

[3] Namavarian N, Rezasoltani A, Rekabizadeh M. [A study on the function of the knee muscles in genu varum and genu valgum (Persian)]. Journal of Modern Rehabilitation. 2014; 8(3):1-9.

[4] Mongashti Joni Y, Fatahi F, Ghanizadeh Hasar N, Hosseinpour E. Effect of genu varum deformity on gluteus medius muscle activity and postural control during single-leg jump-landing. Specific Physical Therapy Journal. 2017; 7(2):79-88. [DOI:10.32598/ptj.7.2.79]

[5] Bakhtiaty AH, Fatemi E, Rezasoltani A. [Genu varum deformity may increase postural sway and falling risk (Persian)]. Koomesh. 2012; 13(3):330-7.

[6] Cote KP, Brunet ME, Gansneder BM, Shultz SJ. Effects of pronated and supinated foot postures on static and dynamic postural stability. Journal of Athletic Training. 2005; 40(1):41-6. [PMID] [PMCID]

[7] Anbarian M, Esmailie H, Hosseini Nejad SE, Rabiei M, Binabaji H. [Comparison of knee joint muscle's activity in subjects with genu varum and the controls during walking and running (Persian)]. Journal of Research in Rehabilitation Sciences. 2012; 8(2):298-309.

[8] Nyland J, Smith S, Beickman K, Armsey T, Caborn DNM. Frontal plane knee angle affects dynamic postural control strategy during unilateral stance. Medicine \& Science in Sports \& Exercise. 2002; 34(7):1150-7. [DOI:10.1097/00005768-200207000-00016] [PMID]

[9] Panahabadi M, Aghayari A, Salari Esker F, Anbarian M. [The effect of genu varum deformity on balance control following postural perturbation in adolescent girls (Persian)]. Scientific Journal of Kurdistan Universiy of Medical Sciences. 2013; 18(2):67-76

[10] Shojaedin SS, Faghihi H. [The relationship between knee varus with dynamic and static postural control in adolescence boys (Persian)]. Journal of Teaching Physical Education. 2014; 2(1):1-7.

[11] Mirmoezzi M, Amini M, Khaledan A, Khorshidi D. [Effect of 8-week of selected aerobic exercise on static and dynamic balance in healthy elderly inactive men (Persian)]. Salmand: Iranian Journal of Ageing. 2016; 11(1):202-9. [DOI:10.21859/sija-1101202]

[12] Kiesel K, Plisky PJ, Voight ML. Can serious injury in professional football be predicted by a preseason functional movement screen? North American Journal of Sports Physical Therapy. 2007; 2(3):147-58. [PMID] [PMCID]

[13] Salehzadeh K, Fathi Rezaee Z, Zamani Sani SH, Sadr Haghighi Kh. [Physical self-concept, body mass index, and physical activity level among college students (Persian)]. Developmental Psychology (Journal of Iranian Psychologists). 2011; 8(29):85-96.

[14] Turkeri C. The effects of 12 weekly salsa training on bmi and static balance. Cukurova University Education Journal. 2014; 44(1):10-22. [DOI:10.14812/cufej.2015.001]

[15] Smith C, Chimera N, Warren W. Association of y balance test reach asymmetry and injury in division I athletes. Medicine \& Science in SportS \& Exercise 2015; 47(1):136-41. [DOI:10.1249/MSS.0000000000000380] [PMID]
[16] Chimera N, Smith C, Warren W. Injury history, sex, and performance on the functional movement screen and $Y$ balance test. Athletic Training 2015; 50(5):475-85. [DOI:10.4085/1062-6050-49.6.02] [PMID] [PMCID]

[17] Johnson F, Leitl S, Waugh W. The distribution of load across the knee. A comparison of static and dynamic measurements. The Journal of Bone and Joint Surgery British Volume. 1980; 62-B(3):346-9. [DOI:10.1302/0301-620X.62B3.7410467]

[18] Thacker SB, Stroup DF, Branche CM, Gilchrist J, Goodman RA, Porte Kelling E. Prevention of knee injuries in sports. A systematic review of the literature. The Journal of Sports Medicine and Physical Fitness. 2003 43(2):165-79. [PMID]

[19] Zemková E, Hamar D. The effect of 6-week combined agility-balance training on neuromuscular performance in basketball players. The Journal of Sports Medicine and Physical Fitness. 2010; 50(3):262-7. [PMID]

[20] McLean SG, Fellin RE, Suedekum N, Calabrese G, Passerallo A, Joy S. Impact of fatigue on gender-based high-risk landing strategies. Medicine \& Science in Sports \& Exercise. 2007; 39(3):502-14. [DOI:10.1249/ mss.0b013e3180d47f0] [PMID]

[21] Peterson ML, Christou E, Rosengren KS. Children achieve adult-like sensory integration during stance at 12 -years-old. Gait \& Posture. 2006 23(4):455-63. [DOI:10.1016/j.gaitpost.2005.05.003] [PMID]

[22] Brown LA, Shumway-Cook A, Woolalacott MH. Attentional demands and postural recovery: The effects of aging. Journal of Gerontology. 1999; 54(4):165-71. [DOI:10.1093/gerona/54.4.M165] [PMID]

[23] Du Pasqier RA, Balnc Y, Sinnreich M, Landis T, Burkhard P. The effect of aging on postural stability: A cross sectional and longitudinal study. Neurophysiologie Clique. 2003; 33(5):213-8. [DOI:10.1016/j.neucli.2003.09.001] [PMID]

[24] Sadeghi H, Mosavi SK, Dizaji E. [Postural stability comparison in various standing positions between healthy young men and those with genu varum (Persian)]. Journal of Research in Rehabilitation Sciences. 2014 10(4):481-91.

[25] Barrett RS, Lichtwark GA. Effect of altering neural muscular and tendinous factors associated with aging on balance recovery using the ankle strategy. Journal of Theoretical Biology. 2008; 254(3):546-54. [DOI:10.1016/j.jtbi.2008.06.018] [PMID]

[26] Desai SS, Shetty GM, Song HR, Lee SH, Kim TY, Hur CY. Effect of foot deformity on conventional mechanical axis deviation and ground mechanical axis deviation during single leg stance and two leg stance in genu varum. Knee. 2007; 14(6):452-7. [DOI:10.1016/j.knee.2007.07.009] [PMID]

[27] Norasteh AA, Hosseini R, Daneshmandi H, Shah Heidari S. [Balance assessment in students with hyperkyphosis and hyperlordosis (Persian)] Sport Medicine (Harakt). 2014; 6(1):57-71.

[28] Raykar R, Tajne K, Palekar T. Effect of forward head posture on static and dynamic balance. World Journal of Pharmaceutical Research. 2018; 7(9):797-808.

[29] Stief F, Bohma H, Schwirtz A, Dussa CU, Doderlein L. Dynamic loading of the knee and hip joint and compensatory strategies in children and adolescents with varus malalignment. Gait \& Posture. 2011; 33(3):490 5. [DOI:10.1016/j.gaitpost.2011.01.001] [PMID]

[30] Junge A, Dvorak J. Soccer injuries: A review on incidence and prevention. Sports Medicine. 2004; 34(13):929-38. [DOI:10.2165/00007256200434130-00004] [PMID]

[31] Anker LC, Weerdesteyn V, van Nes IJ, Nienhuis B, Straatman H, Geurts AC. The relation between postural stability and weight distribution in 
healthy subjects. Gait \& Posture. 2008; 27(3):471-7. [DOI:10.1016/j.gaitpost.2007.06.002] [PMID]

[32] Haim A, Rozen N, Dekel S, Halperin N, Wolf A. Control of knee coronal plane moment via modulation of center of pressure: A prospective gait analysis study. Journal of Biomechanc. 2008; 41(14):3010-6. [DOI:10.1016/j.jbiomech.2008.07.029] [PMID] 